Elsevier required licence: (C) 2018

This manuscript version is made available under the CC-BY-NC-ND 4.0 license http://creativecommons.org/licenses/by-nc-nd/4.0/

The definitive publisher version is available online at https://doi.org/10.1016/j.measurement.2018.01.016 


\title{
Automated Algorithm for Impact Force Identification Using Cosine Similarity Searching
}

\author{
Hamed Kalhori ${ }^{1,2, *}$, Mehrisadat Makki Alamdari ${ }^{3}$, Lin Ye ${ }^{1}$ \\ ${ }^{1}$ Laboratory of smart materials and structures (LSMS), School of Aerospace, Mechanical and Mechatronic \\ Engineering, The University of Sydney, NSW 2006, Australia \\ ${ }^{3}$ School of Civil and Environmental Engineering, University of New South Wales, NSW 2052, Australia
}

\begin{abstract}
A similarity searching technique is adopted to identify the impact force applied on a rectangular carbon fibre-epoxy honeycomb composite panel. The purpose of this study is to simultaneously identify both the location and magnitude of an unknown impact using the measured dynamic response collected by only a single piezoelectric sensor. The algorithm assumes that a set of impact forces are concurrently applied on a set of pre-defined locations. However, the magnitude of all the impact forces except one is considered to be zero. The impact force at all potential locations is then reconstructed through an $l_{2}$-norm-based regularisation via two strategies: even-determined approach and under-determined approach. In an evendetermined approach, the reconstruction process is performed independently for each pair of sensor and potential impact location. However, in an under-determined approach, the captured vibration signal is the superposition of the responses of the simultaneous 'assumed' impacts at the potential locations. Using either approach, a reconstructed impact force is obtained for each potential impact location. The reconstructed impact forces at spurious locations are expected to have zero magnitude as no impact has actually occurred at these locations. However, there might
\end{abstract}

\footnotetext{
${ }^{*}$ To whom all correspondence should be addressed Email: hkal22656@uni.sydney.edu.au, Tel: +61-02-93514798
} 
be some non-zero reconstructed impact forces at spurious locations. Therefore, it is worth designing an automated algorithm capable of detecting the most probable location. Cosine similarity searching is adopted to measure the intensity of the relationship between the reconstructed forces and an impact-like signal with various scale parameters. The largest value of cosine among all reconstructed forces corresponds to the most probable impact location. The results illustrate successful identification of the impact force location and magnitude for both even-determined and under-determined approaches.

Keywords: Composite Panel; Cosine Similarity Searching; Impact Force Identification; Inverse Problem; Piezoelectric.

\section{Introduction}

Composite structures, broadly used in aerospace industry, are vulnerable to damage due to various impact loadings. As a major event in aviation, bird strikes are a substantial and inevitable safety threat to aircrafts [1]. It has been reported that bird strikes can impose more than $\$ 1.2$ billion on the aviation industry for aircraft repairs and delays and cancellations of flights [2].

Common impact-induced failures in composite structures, such as de-bonding of core and skins, delamination of carbon fibre/epoxy laminate skins and core crushing in honeycomb sandwich panels demonstrate the vital need for efficient and low-cost structural health monitoring (SHM) systems. The localisation of damage by identification of impact locations and magnitude can create a speedy SHM system.

Inverse estimation of an impact force would be favourable when information as to the applied force is required but the location of the impact is unknown or inaccessible for direct 
measurement. Inverse algorithms take advantage of impact responses, such as acceleration or strain, which are measurable by typical sensors like accelerometers or strain gauges attached distant from the impact location. Successful application of non-contact sensors using microphone has also been reported in the literature to measure the sound waves induced by impact [3].

Comprehensive identification of an impact force is achieved by determination of both its location and magnitude (force history). De-convolving the response signals from the transfer function of the system is the essential key for reconstructing the impact force history [4]. This approach is typically known as deconvolution. Deconvolution can be performed in both time and frequency domains. However, a review of past research up to 2005 reveals that the vast majority of inverse methods for impact force reconstruction have focused on the frequency domain [5], possibly because of the lower computational cost of the frequency domain compared to the time domain. A frequency domain method was first applied by Bartlett and Flannelly [6] to determine hub forces in a helicopter model. Some research into force identification in the frequency domain can be found in [7-15]. Nowadays, powerful computers have motivated researchers to work in the time domain. The physical behaviour of the system can be better sensed in the time domain [5]. A time-frequency domain method that applies the wavelet transform has also been implemented for force reconstruction [16].

Besides deconvolution methods, neural networks have also attracted much attention for impact identification [17-19]. These techniques entail a considerable amount of training, which limits their application to real large structures [20].

Inverse reconstruction problems are not straightforwardly attainable in reality. The problem stems from the ill-posed nature of the transfer function of the structure. Assume that the impact 
force, $f(t)$, on a structure is mapped to the impact-induced response, $r(t)$, by a linear operator, $\lambda$, as

$$
\lambda[f(t)]=r(t)
$$

Through a perturbation analysis, it can be shown that [5]

$$
\frac{\|\Delta f(t)\|}{\|f(t)\|} \leq \operatorname{cond}(\lambda) \frac{\|\Delta r(t)\|}{\|r(t)\|}
$$

where $\operatorname{cond}(\lambda)$ is the condition number of the linear operator. This number is indicative of the magnification of error in the linear equation. As a result, any tiny perturbation in the data, $\Delta r(t)$, is multiplied by the condition number of $\lambda$, which is usually a very large number. Therefore, the problem must be regularised to avoid a large deviation in the reconstructed force $\Delta f(t)$. It should be noted that $\lambda$ is a convolution operator in impact force problems.

Several $l_{2}$-norm-based regularisation methods, including the Tikhonov, truncated singular value decomposition (TSVD), damped SVD, and iterative regularisation methods, have been proposed to overcome the difficulties of ill-posed problems in the time domain $[21,22]$. Recently, a general sparse methodology based on minimizing $l_{l}$-norm was developed to solve large-scale ill-posed inverse problems for impact force reconstruction [23, 24]. The Wiener filter was also adopted for regularisation of impact force reconstruction problems in the frequency domain $[25,26]$.

In this study, deconvolution is employed to identify the location and magnitude of impact forces exerted on a rectangular carbon fibre-epoxy honeycomb composite sandwich panel. A number of particular locations on the panel are specified as potential places for the occurrence of impact and a single piezoelectric sensor is attached on the underside of the panel to collect the vibration responses. It is assumed that impact forces are simultaneously exerted on all potential 
locations, but the magnitude of all forces except one is zero. The impression behind this scheme is that an impact has occurred at only one of the potential locations. The purpose is to identify the actual impact location as well as its magnitude through a least-squares problem together with regularisation. Two schemes for problem solving are considered: the even-determined approach and the under-determined approach. In the even-determined approach, the identification of impact location and time history is achieved at the same time, however, a large number of equations equal to the number of potential impact locations is required to be solved. In the underdetermined approach, a two-stage procedure is adopted by first localising the impact force using a single equation and then reconstructing the impact force history through a simple deconvolution.

As a result of both schemes, a force history is reconstructed for each potential impact location. However, chances are high that non-zero reconstructed forces appear at spurious locations where no impact has actually occurred at these locations. Cosine similarity searching is utilised to find the actual impact location based on measuring the cosine of the angle between the reconstructed force vectors and an impact-like vector. It is demonstrated that the reconstructed force at the actual location is characterised by the highest similarity index. Several case studies using a panel with eight potential impact locations are investigated.

\section{Inverse Problem}

For an impact force problem, Equation (1) can be expressed by using a convolution operator as [27]

$$
\int_{0}^{t} g(\vartheta, \varsigma, t-\tau) f(\vartheta, \tau) d \tau=r(\varsigma, t)
$$


where $g(\vartheta, \varsigma, t-\tau)$ is the transfer function in the time domain between impact location $\vartheta$ and measurement point $\varsigma$. By applying Riemann's approximation, Equation (3) is given by

$$
\sum_{i=0}^{n-1} g_{i+1} f_{n-i}=r_{n} \quad, \quad(n=1, \ldots, p)
$$

where $r_{n}(n=1, \ldots, p)$ is the response at time $t_{n}=n . \Delta t, \Delta t$ is the sampling time and $p$ is the number of samples. Equation (4) can be expressed in matrix form as

$$
G F=R,
$$

where,

$$
G=\left[\begin{array}{ccccc}
g_{1} & 0 & \ldots & \ldots & 0 \\
g_{2} & g_{1} & \ddots & \ddots & \vdots \\
g_{3} & g_{2} & \ddots & \ddots & \vdots \\
\vdots & \vdots & \ddots & \ddots & 0 \\
g_{p} & g_{p-1} & \cdots & \cdots & g_{1}
\end{array}\right], \quad F=\left[\begin{array}{c}
f_{1} \\
f_{2} \\
f_{3} \\
\vdots \\
f_{p}
\end{array}\right], \quad R=\left[\begin{array}{c}
r_{1} \\
r_{2} \\
r_{3} \\
\vdots \\
r_{p}
\end{array}\right] .
$$

Assuming a number of impact forces at different locations $F_{i}(i=1 \ldots X)$ simultaneously applied to a structure, the corresponding dynamic strain signal at a given single measurement point $R$ is a superposition of the responses caused by each single force.

$$
G^{1} F_{1}+G^{2} F_{2}+\ldots+G^{X} F_{X}=\sum_{i=1}^{X} G^{i} F_{i}=R
$$

where $G^{i}$ is the transfer function between the force location $i$ and the sensor location. Equation (7) is written in matrix-vector form as

$$
\left[\begin{array}{llll}
G^{1} & G^{2} & \cdots & G^{X}
\end{array}\right]\left[\begin{array}{c}
F_{1} \\
F_{2} \\
\vdots \\
F_{X}
\end{array}\right]=[R]
$$


where $X$ is the number of impact locations. For a problem with one impact location, Equation (8) represents an even-determined problem and is the same as Equation (5). However, for a problem with more than one impact location, Equation (8) produces an under-determined problem. For simplicity, Equation (8) is represented by $G F=R$. The solution is then obtained using the leastsquares problem as

$$
\min \|G F-R\|_{2}^{2}
$$

Since $R$ is practically contaminated by experimental errors and $G$ is a matrix with a very large condition number, the problem must be regularised. Tikhonov regularisation seeks a good approximation of $F$ by replacing Equation (9) with a penalised least-squares problem of the form

$$
\min \left\{\|G F-R\|_{2}^{2}+\beta\|I F\|_{2}^{2}\right\}
$$

where $I$ is the identity matrix and $\beta \geq 0$ is the regularisation parameter, which can be determined by the generalised cross-validation (GCV) method [21]. The optimal regularisation parameter can also be determined as a solution of a maximisation problem [28].

\section{Cosine Similarity}

As a fundamental principle of the presented study, it is assumed that a number of impact forces are synchronously applied at the established impact locations as shown in Figure 1, but the magnitude of all forces except one is zero, indicating that the impact has in fact occurred at one location only.

After reconstruction of impact forces at all possible locations, it is expected that all forces except the one at the true impact location will be of very low amplitude (around zero). However, this is not the case in most of the analyses performed in this study. Previous studies have 
proposed some qualitative and quantitative assessment measures based on the characteristics of the reconstructed forces, including the shape, the maximum amplitude of the first peak (if any), and the momentum change of the impactor [29]. However, the qualitative analysis required a manual decision-making process.

From a qualitative viewpoint, a typical impact force time history is expected to include localised narrow-band high-energy components with smooth temple shapes with or without multiple reflections. Furthermore, since an impact force is totally compressive, there should be no negative portion in the reconstructed impact force. Besides, in the case of existing multiple reflections or local peaks in impact force as a result of a normal free strike, the first peak normally has a higher energy than the next peaks. Figure 2 shows some sample impact profiles produced by different impactors on the panel. Based on these characteristics, application of the cosine similarity can be quite advantageous for identifying these time-localised events and scalelocalised components.

Mathematically, a similarity metric can be used to depict the similarity level for several discrete time series in temporal data mining. The similarity-searching technique can be applied to find the most similar time series, in which the distance between discrete time series is considered as a similarity metric. Cosine similarity is a unique type of similarity measurement in which the cosine of the angle between two non-zero vectors is utilized to calculate the error between the two vectors. Two vectors with the same orientation have a cosine similarity of 1 , and two vectors at $90^{\circ}$ have a similarity of 0 . The magnitude of the vectors is not considered in cosine similarity. In rectangular coordinates, the cosine similarity of the two vectors $p=\left(p_{1}, p_{2}, \ldots, p_{n}\right)$ and $q=\left(q_{1}, q_{2}, \ldots, q_{n}\right)$ is given by 


$$
M(p, q)=\frac{p \cdot q}{\|p\|\|q\|}=\frac{\sum_{i=1}^{n} p_{i} q_{i}}{\sqrt{\sum_{i=1}^{n} p_{i}^{2}} \sqrt{\sum_{i=1}^{n} q_{i}^{2}}} .
$$

In this study, the similarity of a reconstructed force at a potential impact location is measured compared with a half-sine vector with various scale parameters $a$. Basically, the global shape of an impact force is expected to be similar to a half-sine signal. As pointed out in [30], even in the presence of damage, the global shape of an impact force will still be analogous to a half-sign signal with local fluctuations. Scale parameter controls the width of the half-sine. The smaller the scale factor, the more compressed the half-sine. Conversely, the larger the scale, the more stretched the half-sine. The half-sine signal is defined as

$$
p= \begin{cases}\sin \left(\frac{\pi}{a} t\right) & 0<t<a \\ 0 & a<t<T\end{cases}
$$

where $T$ represents the time window. A similarity index $\delta$ is then introduced as

$$
\delta=\max \|M(a)\|
$$

where $\delta$ denotes the maximum of $M$ for the entire range of the scale. It is expected that $\delta$ reaches its maximum value at the true source location of impact among all the other possible locations, as

$$
\Delta=\arg \max \left(\delta_{i}\right), \quad i=1: X
$$

where $\Delta$ corresponds to the location of the source of impact and $X$ is the number of potential impact locations. This property is adopted in the present study to identify the source location of impact.

\section{Experimental Set-up and Procedure}


This section describes the experimental work undertaken to investigate the impact force identification strategy on a composite panel. A carbon fibre composite sandwich panel (600 mm in length and $400 \mathrm{~mm}$ in width) encompassing 4 woven plies in a quasi-isotropic lay-up $[ \pm 45$, $0 / 90]_{\mathrm{s}}$ with a nominal thickness of $0.88 \mathrm{~mm}$ and a honeycomb core of $20 \mathrm{~mm}$ thickness was used in the experiment. The manufacturing process of the composite panel was described in detail in [31-33]. The panel was clamped at two opposite edges while the other two edges were freesupported. The panel was instrumented with a single circular PIC 151 piezoceramic sensor (10 $\mathrm{mm}$ diameter and $1 \mathrm{~mm}$ thickness) which was surface-bonded on the underside of the specimen using Loctite Super Glue at the point shown in Figure 1.

A circular piezoceramic sensor is able to measure the strain invariant $\varepsilon_{T}=\varepsilon_{r}+\varepsilon_{\theta}$, where $\varepsilon_{r}$ and $\varepsilon_{\theta}$ are radial and circumferential strain components. The strain invariant $\varepsilon_{T}$ is related to the experimentally collected output voltage $V_{\text {out }}$ by

$$
\varepsilon_{T}=\psi V_{\text {out }}
$$

where $\psi$ is a constant depending on the thickness and mechanical properties of the piezoceramic sensor and the bonding effects of Loctite Super Glue [34].

A grid was drawn on the specimen, as shown in Figure 1, to specify 8 possible locations of impact, evenly distributed on upper face of the panel. Impact force was applied using a modal hammer (Meggitt's Endevco, 2303) and the signals of the piezoelectric sensor and the modal hammer were collected by an oscilloscope (Tektronix DPO 4034B). Signal acquisition was set to begin as soon as the impact force produced by the hammer exceeded $20 \mathrm{~N}$. Signal acquisition was performed at a sampling rate of $50 \mathrm{kHz}$ and captured signals for the duration of $10 \mathrm{~ms}$. Figure 3 depicts the experimental set-up.

As previously explained, identification of impact force through deconvolution requires 
knowledge about dynamic characteristics, i.e. the transfer function of the structure. To generate the transfer function between each potential impact location (8 locations) and a PZT sensor, a set of known impact forces were first applied at each impact location and the resultant dynamic strains were collected by a single piezoceramic sensor. A complete procedure for establishing the transfer function can be found in previous studies [29, 35-37]. An impact force with unknown amplitude and location was then applied at one of the potential locations. The purpose was to discover the impact magnitude together with the location of impact using the response of only one sensor installed underneath the panel.

In this study, it is assumed that the structural damping is negligible and the changes in the system characteristics before and after the impact are ignorable. In addition, it is assumed that the structure is at rest before an external load is applied. It is also considered that there are no concurrent multiple impacts and only a single impact force is applied to the structure at each time.

\section{Results and Discussion}

As mentioned previously, two different approaches were considered to identify an unknown impact force. In the first approach, an even-determined problem was considered and the reconstruction problem was performed individually for each pair of sensor and potential impact location. In the second approach, an under-determined problem was targeted and all impact forces were simultaneously reconstructed. Eight case studies were carried out. In each case, the actual impact was applied at one of the 8 potential locations. As an example, in the first case study, the impact was actually applied at location 1 and the forces at all potential locations were reconstructed. This scenario was performed for all other potential locations. 
The automated process of impact force identification using the cosine similarity index, elaborated in Section 3, was followed to identify the true impact location from 8 potential locations.

\subsection{Even-Determined Approach}

In an even-determined approach, the reconstruction process was carried out independently for each impact location (see Equation (5)). As a case study, the actual impact was considered to occur at location 6 . The solution strategy proceeded assuming that an impact had occurred at location 1 . The impact force was then reconstructed at that location implementing the collected dynamic response and the transfer function between sensor and impact location $1, G^{1}$. This procedure was repeated for impact locations 2 to 8 utilising $G^{2}$ to $G^{8}$, respectively. Figure 4 provides a schematic of the problem. Figure 5 illustrates the reconstructed impact forces for all the 8 potential locations. In Figure 5, 'Actual' represents the actual force applied at each location.

By qualitatively investigating the reconstructed impact forces, it can be readily realised that the actual impact has occurred at location 6 as shown in Figure 5(f). The other 7 locations cannot be a true impact force since they do not have the characteristics of an impact force e.g. being positive and smooth, having a global half-sine shape and having zero value at $t=0$.

The purpose of this study is to identify the true impact location in a fully-automated manner, therefore, the cosine similarity index is calculated to investigate which of the 8 reconstructed impact forces has the highest similarity and correlation with a half-sine signal. As no information about the duration of impact, hence, its frequency, is available beforehand, therefore, the shape of the half-sine signal is controlled by a scale parameter to check the similarity for a continuum 
range of scale values.

For the actual impact location 6 , the cosine similarity index was calculated for a scale value ranging from 1 to 104 . Figure 6 presents the cosine similarity between the reconstructed force at location 6 and the half-sine signal with various scales. A maximum similarity is obtained for scale value of 61 . Figure 7 compares the actual impact force at location 6 and the half-sine signal with scale of 61 . A very good correlation between these two signals can be observed which implies the fact that for a given true impact force, there should be a scale value that provides the maximum similarity. It should be emphasized that no a priori information about the optimum candidate of the scale value is available beforehand. Basically, for each potential impact location, by changing the scale value, the maximum achievable similarity index is obtained and then these maximum values are further compared according to Equation (14) to find the true impact location since it is expected that the true impact location results in maximum similarity index. It is obvious that the optimum scale that produces maximum similarity for a specific impact location might differ from the one obtained for another location.

On this basis, the process of impact force identification was repeated for 8 different cases where in each case one of the 8 locations is the true impact force location. The similarity index was obtained using Equation (13) and the results are presented in Figure 8. As indicated, the proposed index can successfully localise the true impact location for all 8 cases investigated.

Although, very promising force identification results were obtained considering an evendetermined problem, this process is not very efficient since the problem needs to be solved more than once, depending on the number of potential locations e.g. 8 in this study. Therefore, a more efficient approach of solving this problem is to consider an under-determined problem. The results using this approach is presented in the next section. 


\subsection{Under-Determined Approach}

A more efficient approach of impact force identification is to simultaneously identify all the potential impact forces and disregard the spurious locations based on the proposed similarity index. The under-determined approach is based on deconvolution of a matrix equation composed of linear superposition of responses to impact forces at different locations (see Equation (8)). Unlike the even-determined approach, the impact forces at all potential locations were reconstructed concurrently through a single equation. Figure 9 provides a schematic of the problem.

Figure 10 illustrates the time history of the reconstructed impact forces while the true impact location is at location 6. Qualitative examination of this figure reveals that location 6 is of the highest chance to be an impact location, however, unlike the previous approach, the reconstructed force has higher discrepancy with the true impact force, see Figure 10 (f), compared to the case when an even-determined problem was solved, see Figure 5 (f). The reason for this poor reconstruction is simply due to the high indeterminacy in the system. Basically, in the previous approach, the problem of impact force identification was solved eight different times for each pair of sensor and potential impact location. It means that Equation (8) is converted to eight linear equations to determine the eight impact forces separately by solving each linear equation. However, in this approach, the impact forces at eight potential locations are simultaneously identified considering Equation (8) as a matrix equation incorporating the impact forces at all potential locations. Therefore, all the impact forces are calculated at the same time.

For all possible impact locations, the second approach is carried out and the similarity index is calculated based on Equation (13). The results are presented in Figure 11. As illustrated, the location of the true impact force can be successfully identified in all the cases which 
demonstrates the fact that the proposed method can reliably localise the impact force from the potential candidate locations by solving only one equation. After successful localisation, the impact force can be quantified by solving an even-determined problem considering the transfer function between the sensor and the identified impact location. This step is referred to as finetuning, resulting in accurate reconstruction of the force time-history.

\section{Concluding remarks}

The paper investigated the process of impact force identification in a fully automated and efficient way implementing a single piezoelectric sensor. Two different approaches were considered to reconstruct the impact forces applied at pre-defined potential locations either by solving an under-determined problem or solving an even-determined problem. Having the impact forces reconstructed for all potential locations, a cosine similarity index was introduced to automatically localise the true impact location. The approach was applied on a rectangular carbon fibre-epoxy honeycomb composite panel consisting of 8 potential impact locations. It was revealed that both even-determined and under-determined approaches could reliably localise the true impact location, however, solving an under-determinant problem was more efficient than solving an even-determinant problem. The promising results from this work can open opportunities for application of the method for force identification in real-life applications. 


\section{References}

[1] V. Bheemreddy, K. Chandrasekhara, Study of bird strikes using smooth particle hydrodynamics and stochastic parametric evaluation, Journal of Aircraft, 49 (2012) 1513-1520.

[2] J. Allan, A heuristic risk assessment technique for birdstrike management at airports, Risk analysis, 26 (2006) 723-729.

[3] S. Atobe, S. Nonami, N. Hu, H. Fukunaga, Identification of impact force acting on composite laminated plates using the radiated sound measured with microphones, Journal of Sound and Vibration, 405 (2017) 251-268.

[4] E. Jacquelin, A. Bennani, P. Hamelin, Force reconstruction: analysis and regularization of a deconvolution problem, Journal of Sound and Vibration, 265 (2003) 81-107.

[5] F.E. Gunawan, Impact force reconstruction using the regularized Wiener filter method, Inverse Problems in Science and Engineering, (2015) 1-26.

[6] F. Bartlett, W. Flannelly, Model verification of force determination for measuring vibratory loads, Journal of the American Helicopter Society, 24 (1979) 10-18.

[7] R. Adams, J.F. Doyle, Multiple force identification for complex structures, Experimental Mechanics, 42 (2002) 25-36.

[8] J.F. Doyle, An experimental method for determining the dynamic contact law, Experimental Mechanics, 24 (1984) 10-16.

[9] J.F. Doyle, Further developments in determining the dynamic contact law, Experimental mechanics, (1984) 265-270.

[10] J.F. Doyle, Determining the contact force during the transverse impact of plates, Experimental Mechanics, 27 (1987) 68-72.

[11] H. Inoue, M. Kamibayashi, K. Kishimoto, T. Shibuya, T. Koizumi, Numerical Laplace transformation and inversion using fast Fourier transform, JSME international journal. Ser. 1, Solid mechanics, strength of materials, 35 (1992) 319-324.

[12] H. Inoue, K. Kishimoto, T. Shibuya, K. Harada, Regularization of Numerical Inversion of the Laplace Transform for the Inverse Analysis of Impact Force, JSME International Journal Series A, 41 (1998) 473-480.

[13] Y. Liu, W.S. Shepard, Dynamic force identification based on enhanced least squares and total least-squares schemes in the frequency domain, Journal of sound and vibration, 282 (2005) 37-60.

[14] S. Ödeen, B. Lundberg, Prediction of impact force by impulse response method, International Journal of Impact Engineering, 11 (1991) 149-158.

[15] E. Parloo, P. Verboven, P. Guillaume, M. Van Overmeire, Force identification by means of in-operation modal models, Journal of Sound and Vibration, 262 (2003) 161-173.

[16] J.F. Doyle, A wavelet deconvolution method for impact force identification, Experimental mechanics, 37 (1997) 403-408.

[17] S.E.W. Akhavan, K. Chandrashekhara, Farhad, Prediction of impact contact forces of composite plates using fiber optic sensors and neural networks, Mechanics of Composite Materials and Structures, 7 (2000) 195-205.

[18] B.W. Jang, Y.G. Lee, J.H. Kim, Y.Y. Kim, C.G. Kim, Real-time impact identification algorithm for composite structures using fiber Bragg grating sensors, Structural Control and Health Monitoring, 19 (2012) 580-591.

[19] K. Worden, W. Staszewski, Impact location and quantification on a composite panel using neural networks and a genetic algorithm, Strain, 36 (2000) 61-68. 
[20] J. Park, S. Ha, F.-K. Chang, Monitoring impact events using a system-identification method, AIAA journal, 47 (2009) 2011-2021.

[21] P.C. Hansen, Regularization tools: A Matlab package for analysis and solution of discrete ill-posed problems, Numerical algorithms, 6 (1994) 1-35.

[22] P.C. Hansen, Rank-deficient and discrete ill-posed problems: numerical aspects of linear inversion, Siam, 1998.

[23] B. Qiao, X. Zhang, J. Gao, X. Chen, Impact-force sparse reconstruction from highly incomplete and inaccurate measurements, Journal of Sound and Vibration, 376 (2016) 72-94.

[24] B. Qiao, X. Zhang, J. Gao, R. Liu, X. Chen, Sparse deconvolution for the large-scale illposed inverse problem of impact force reconstruction, Mechanical Systems and Signal Processing, (2016).

[25] F.E. Gunawan, H. Homma, Y. Kanto, Two-step B-splines regularization method for solving an ill-posed problem of impact-force reconstruction, Journal of Sound and Vibration, 297 (2006) 200-214.

[26] H. Inoue, J.J. Harrigan, S.R. Reid, Review of inverse analysis for indirect measurement of impact force, Applied Mechanics Reviews, 54 (2001) 503.

[27] D.J. Inman, Engineering vibration, Prentice-Hall, 2001.

[28] N. Hu, S. Matsumoto, R. Nishi, H. Fukunaga, Identification of impact forces on composite structures using an inverse approach, Structural Engineering and Mechanics, 27 (2007) 409-424.

[29] H. Kalhori, L. Ye, S. Mustapha, Inverse estimation of impact force on a composite panel using a single piezoelectric sensor, Journal of Intelligent Material Systems and Structures, (2016) $1045389 X 16657424$.

[30] S. Atobe, S. Sugimoto, N. Hu, H. Fukunaga, Impact damage monitoring of FRP pressure vessels based on impact force identification, Advanced Composite Materials, 23 (2014) 491-505.

[31] S. Mustapha, L. Ye, Leaky and non-leaky behaviours of guided waves in CF/EP sandwich structures, Wave Motion, 51 (2014) 905-918.

[32] S. Mustapha, L. Ye, Propagation behaviour of guided waves in tapered sandwich structures and debonding identification using time reversal, Wave Motion, (2015).

[33] S. Mustapha, L. Ye, D. Wang, Y. Lu, Debonding detection in composite sandwich structures based on guided waves, AIAA journal, 50 (2012) 1697-1706.

[34] S.M. Peelamedu, N.G. Naganathan, R.V. Dukkipati, J. Srinivas, Impact identification for a metallic plate using distributed smart materials, Smart materials and structures, 14 (2005) 449.

[35] H. Kalhori, L. Ye, S. Mustapha, J. Li, B. Li, Reconstruction and Analysis of Impact Forces on a Steel-Beam-Reinforced Concrete Deck, Experimental Mechanics, (2016) 1-12.

[36] H. Kalhori, L. Ye, Z. Li, B. Li, Identification of Location and Magnitude of Impact Force on a Composite Sandwich Structure With Lattice Truss Core, ASME 2015 International Mechanical Engineering Congress and Exposition, American Society of Mechanical Engineers, 2015, pp. V001T001A023-V001T001A023.

[37] H. Kalhori, L. Ye, S. Mustapha, J. Li, Impact force reconstruction on a concrete deck using a deconvolution approach, 8th Australasian Congress on Applied Mechanics: ACAM 8. Barton, ACT: Engineers Australia, (2014) 763-771. 


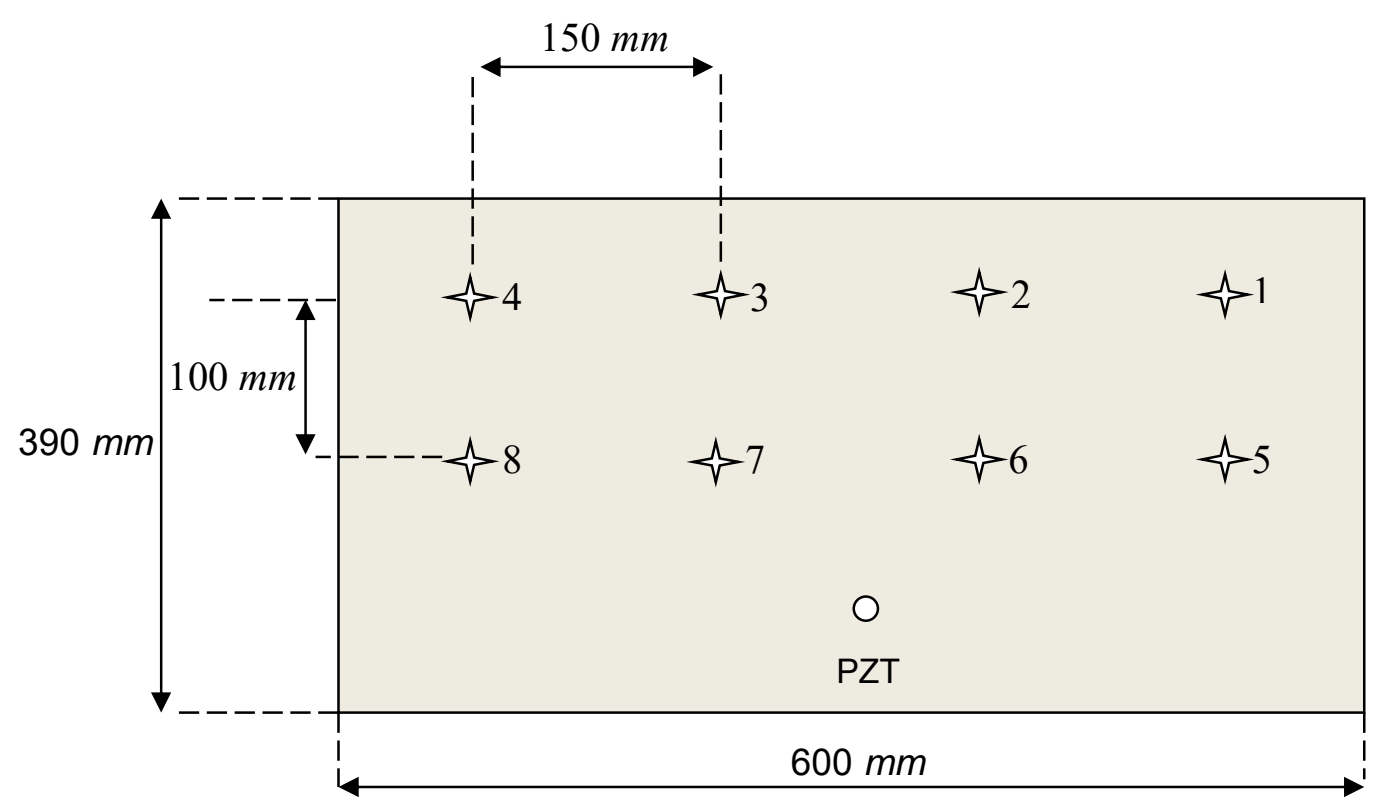

Figure 1. Location of the piezoceramic disk and the potential impact forces. 


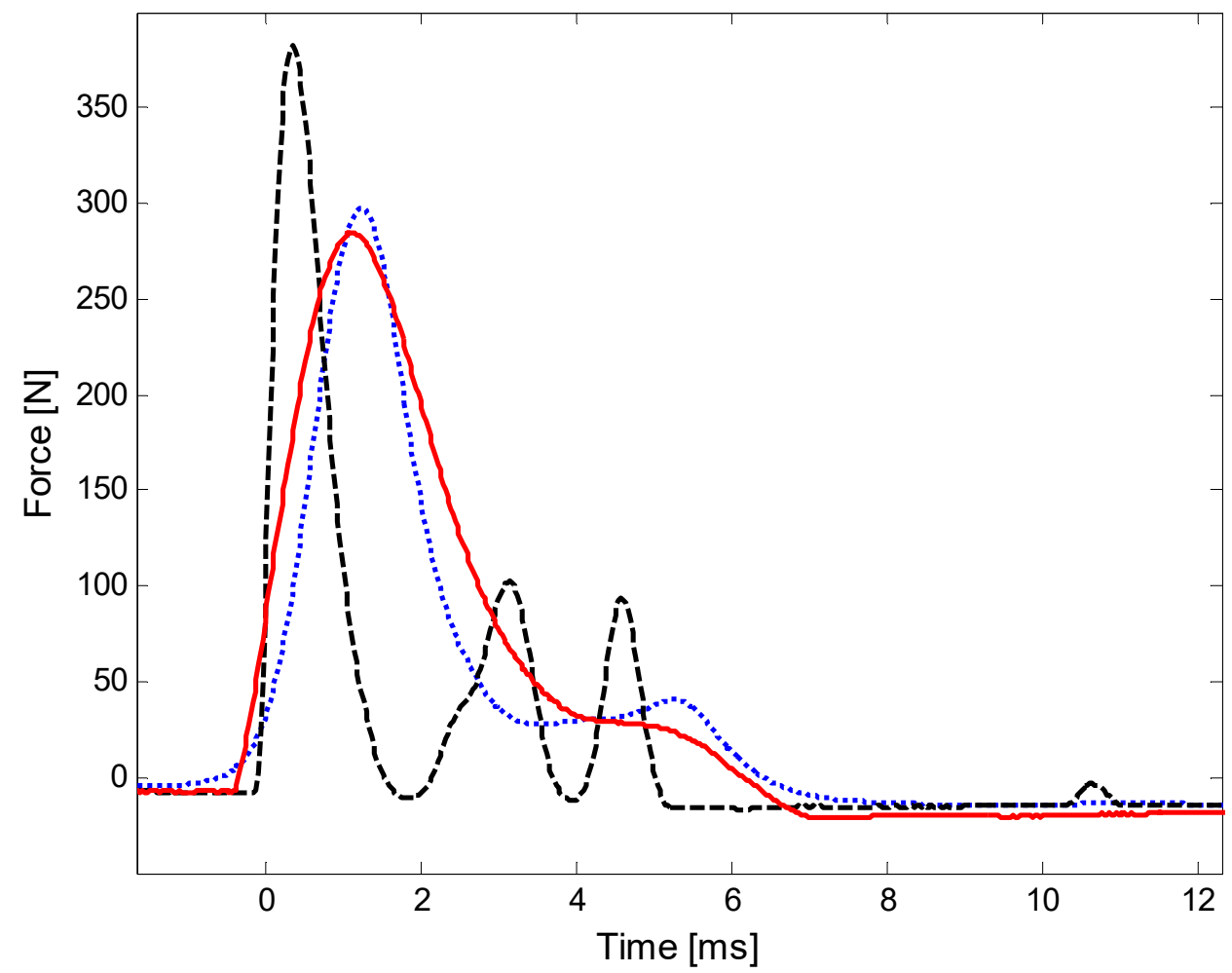

Figure 2. Representative impact profiles applied by various impactors on the panel.

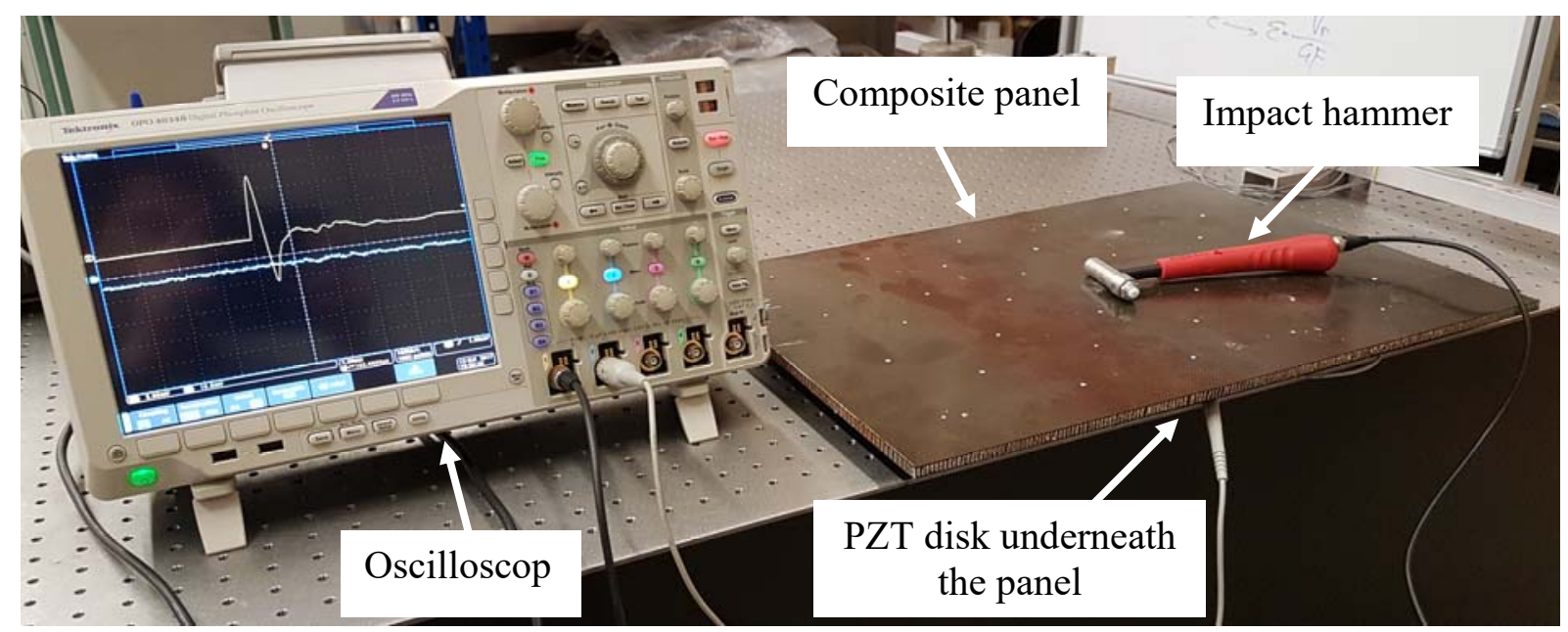

Figure 3. The experimental set-up including the composite panel, impact hammer, oscilloscope, and PZT disk. 


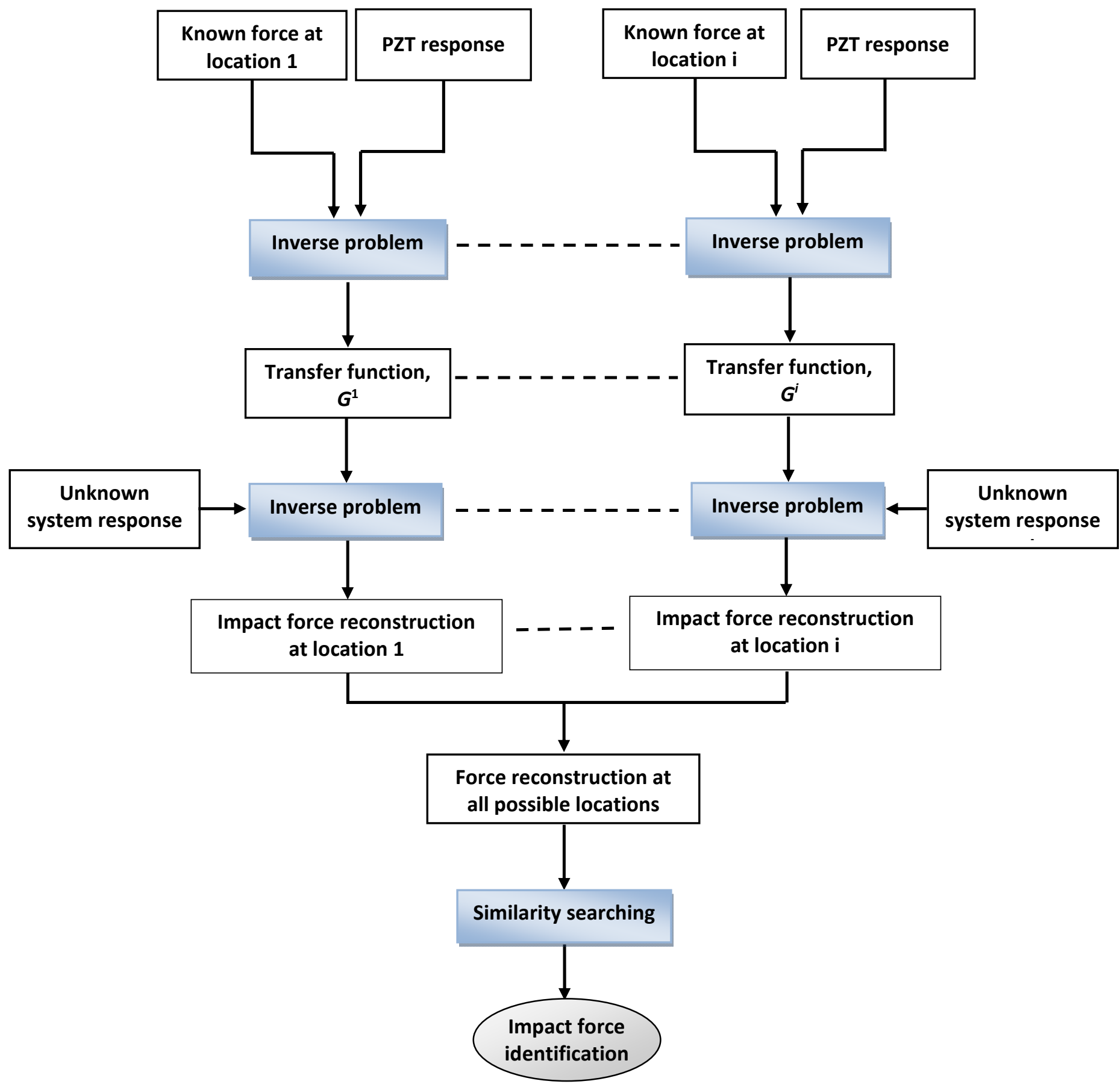

Figure 4. Schematic of impact force identification algorithm using the even-determined approach. 

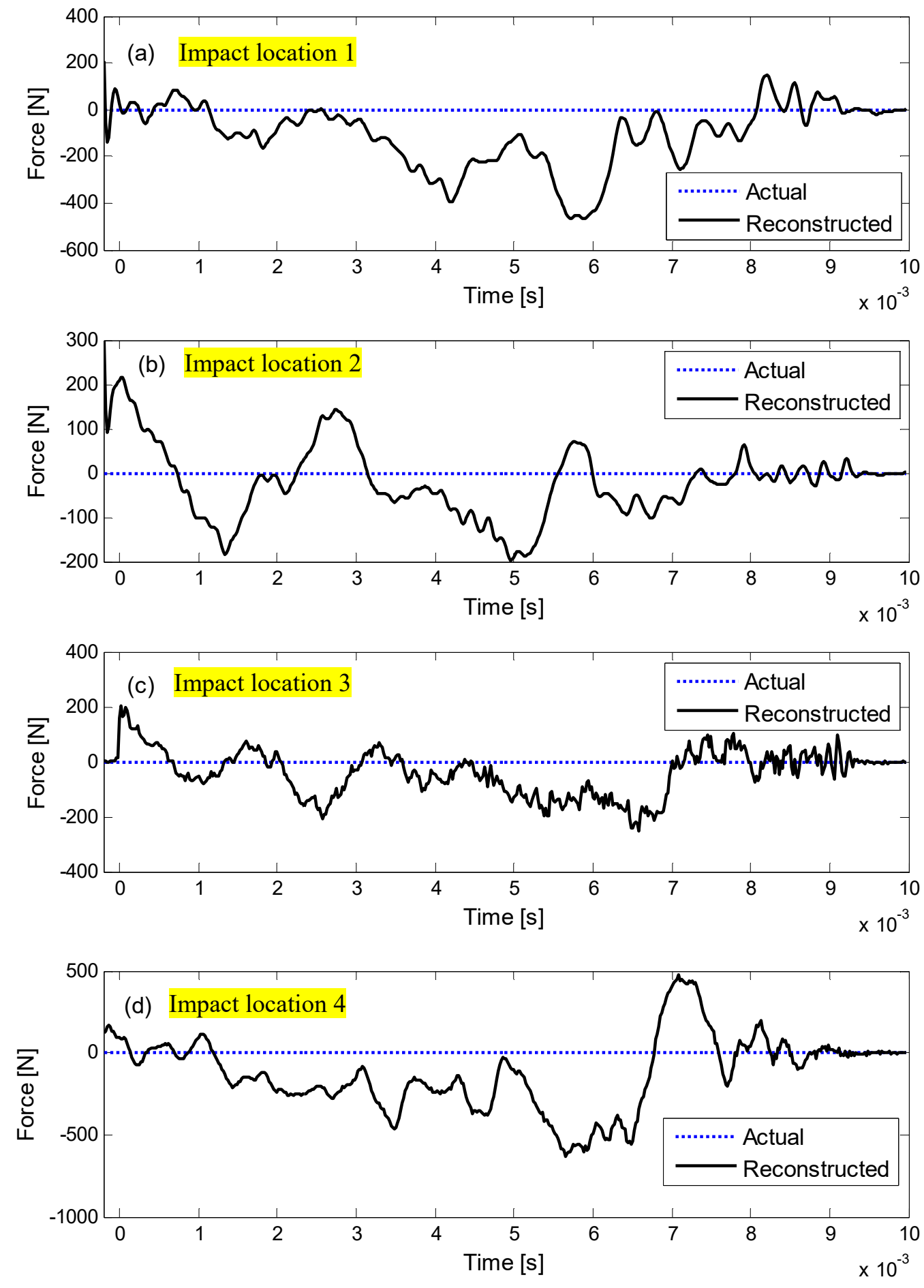

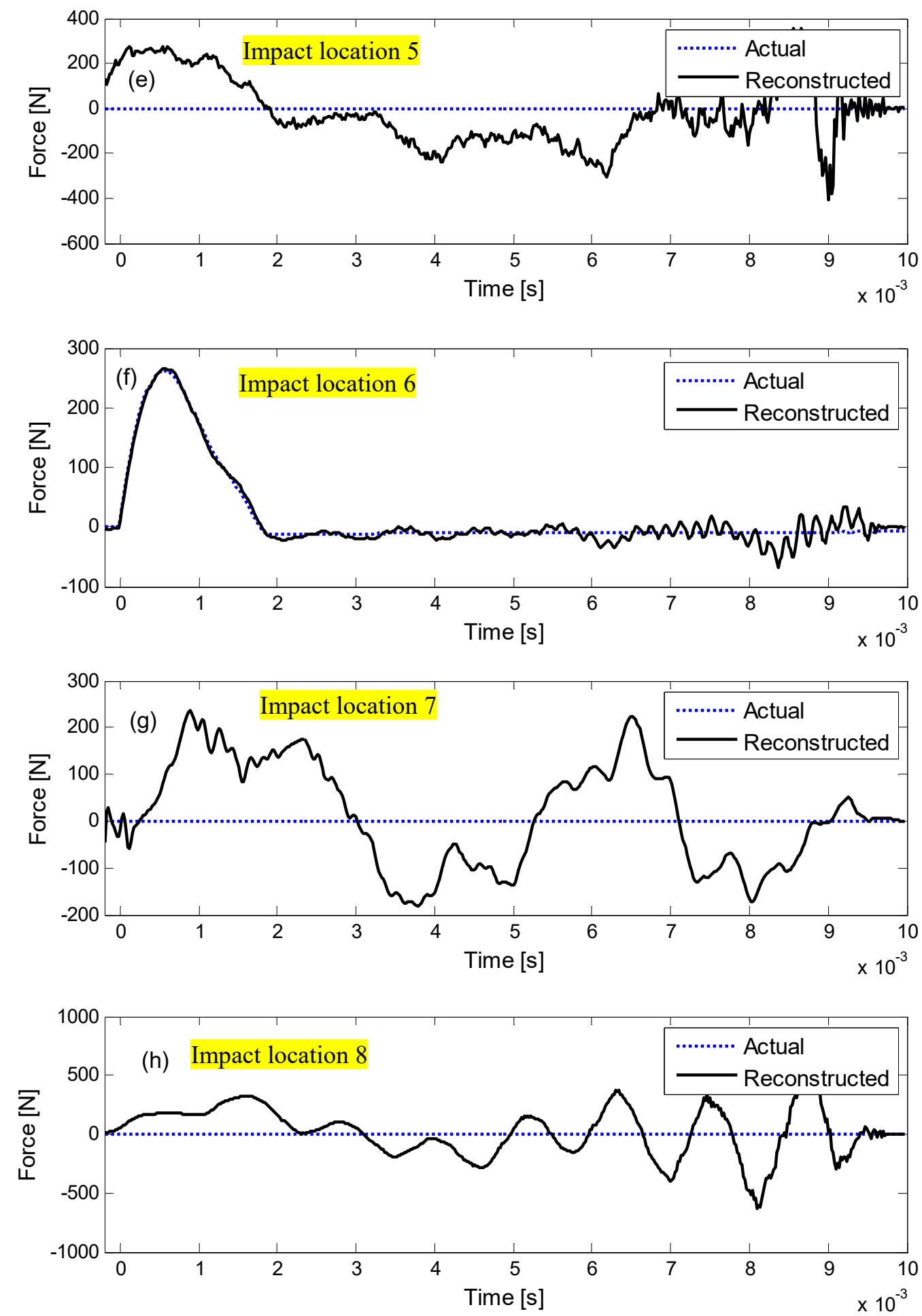

Figure 5. The reconstructed impact forces using the even-determined approach while the true impact location is location 6 . 


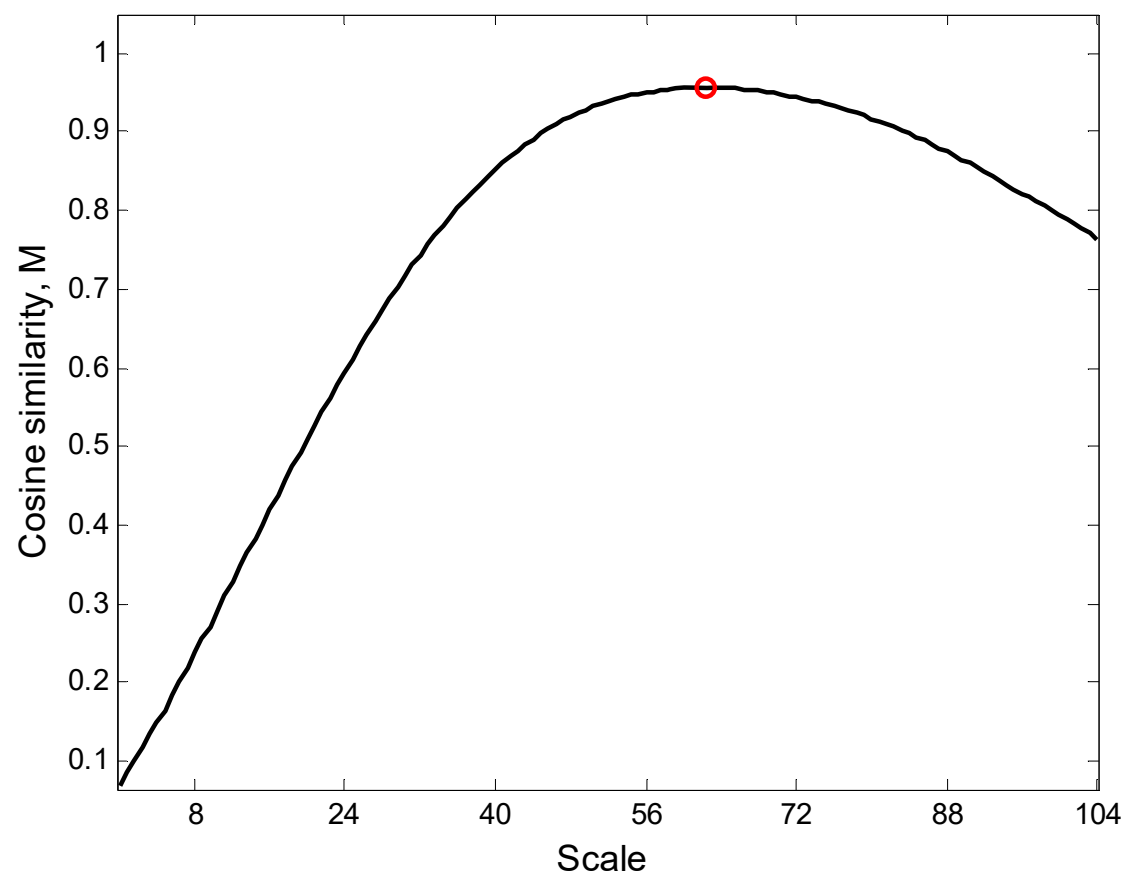

Figure 6. Cosine similarity between the reconstructed force at location 6 and the half-sine signal with various scales.

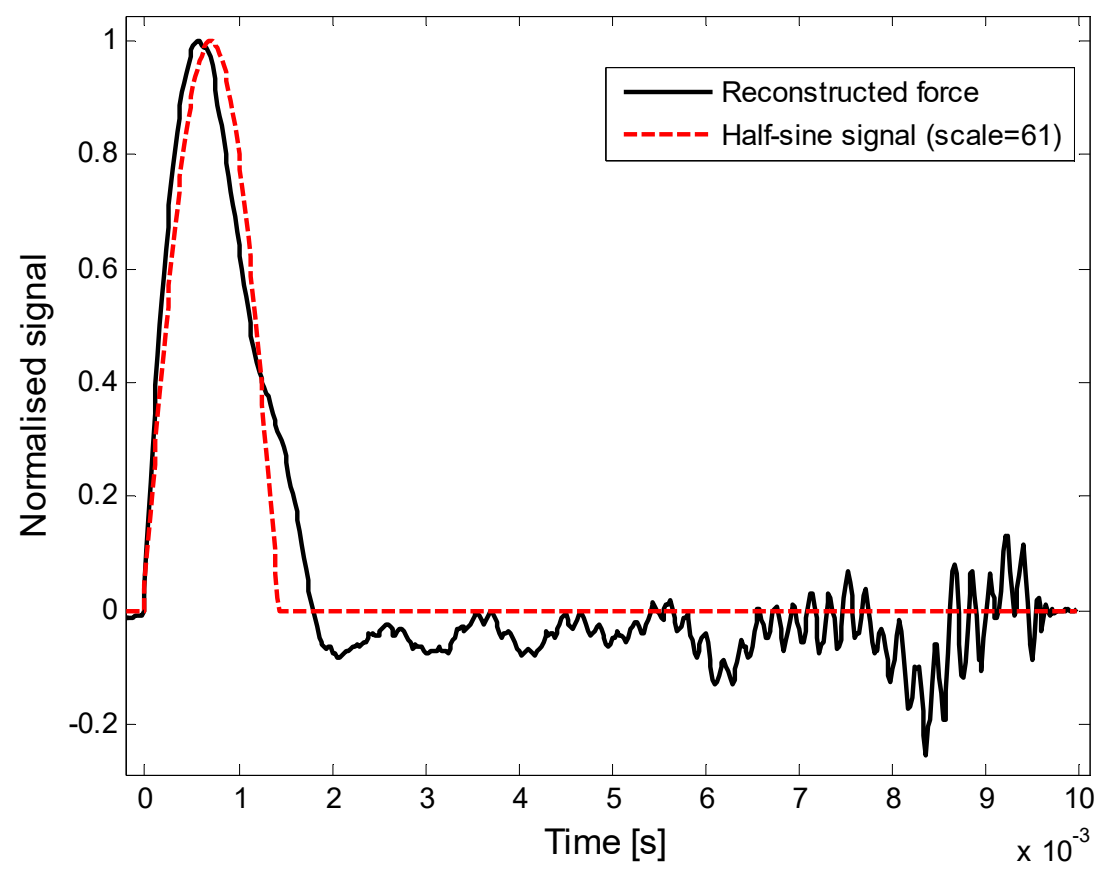

Figure 7. Comparison of the reconstructed force at location 6 with the half-sine signal with scale value of 61 . 

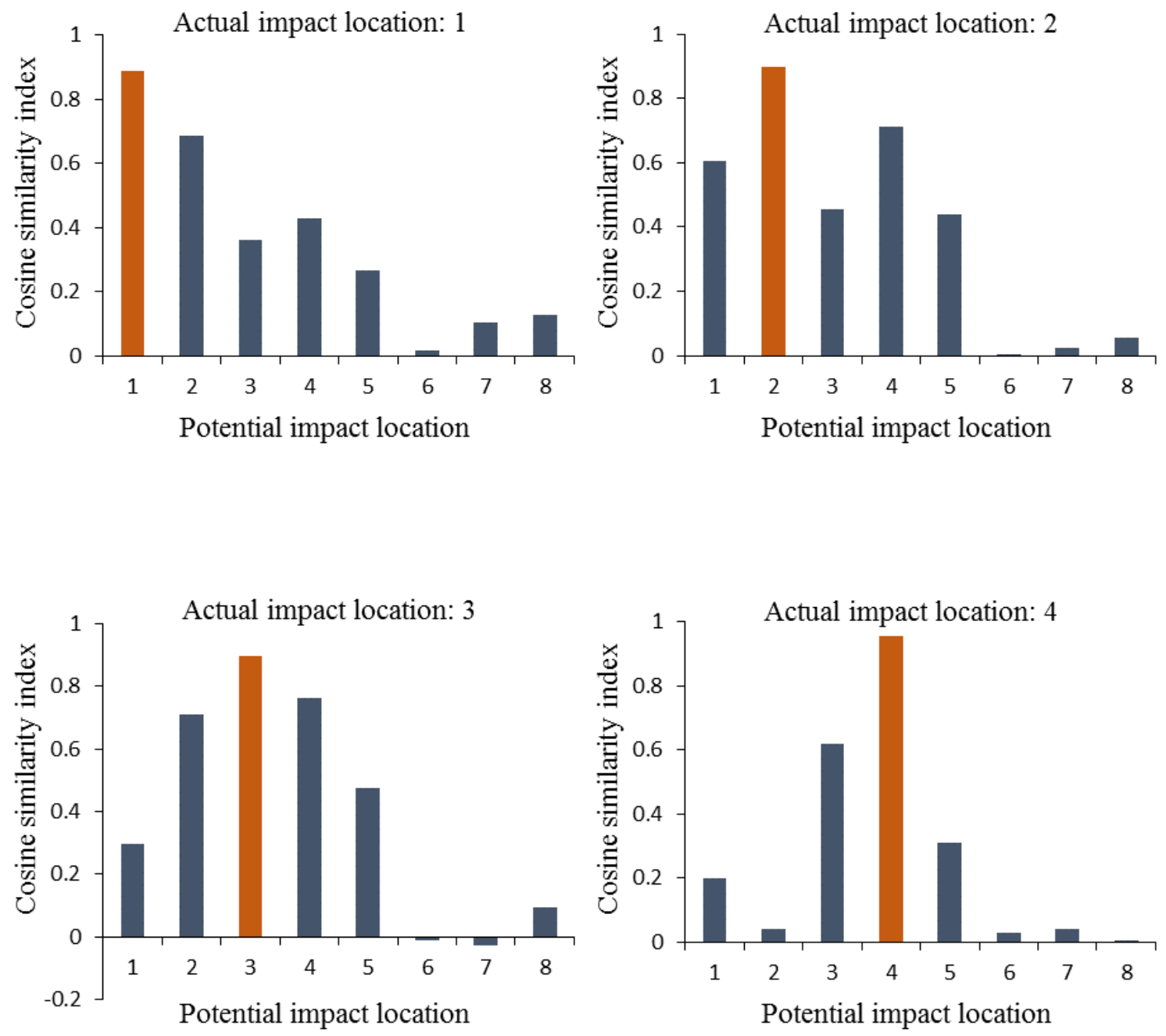

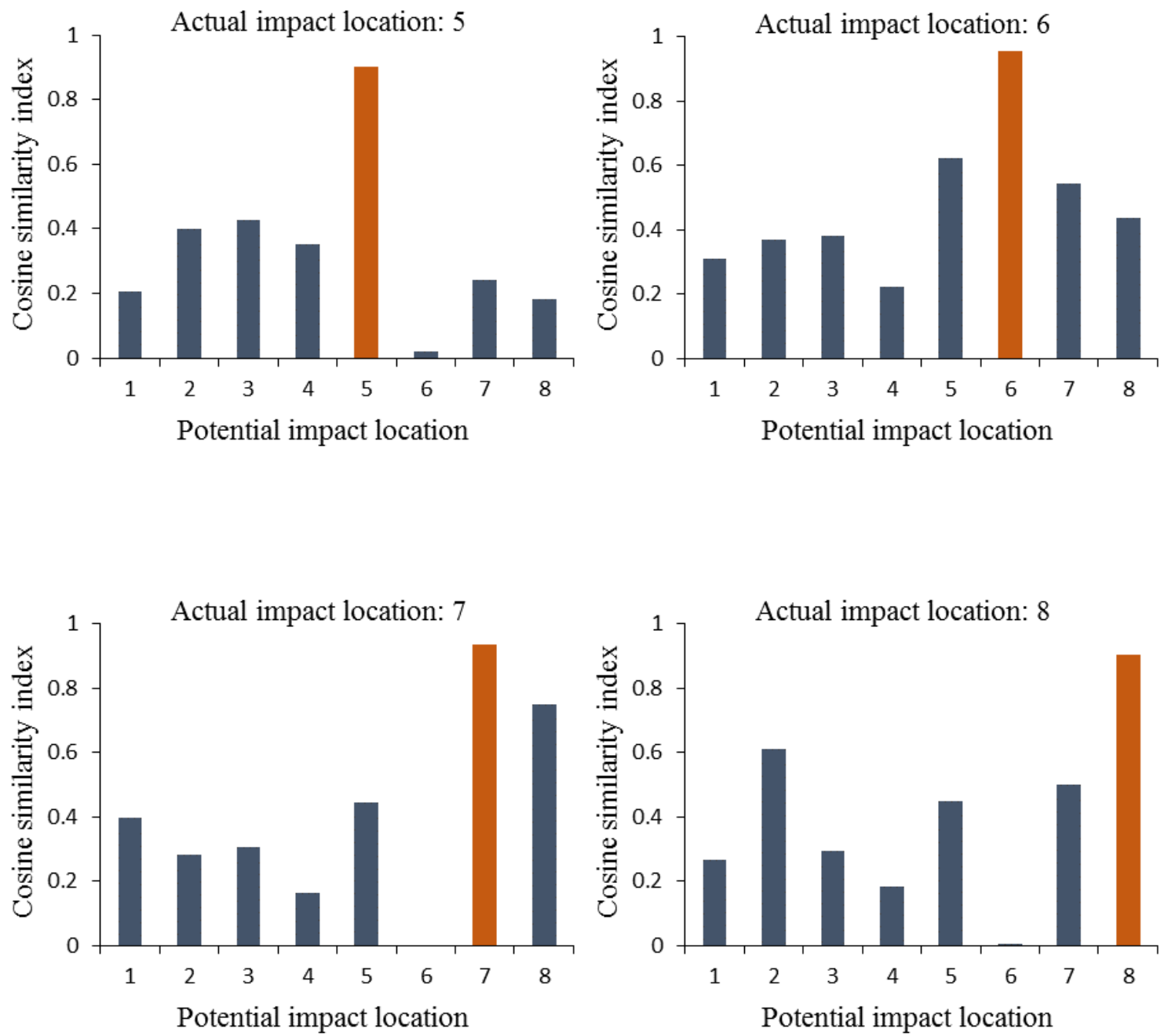

Figure 8 . The cosine similarity indices for the all investigated cases when the impact forces were reconstructed using the even-determined approach. 


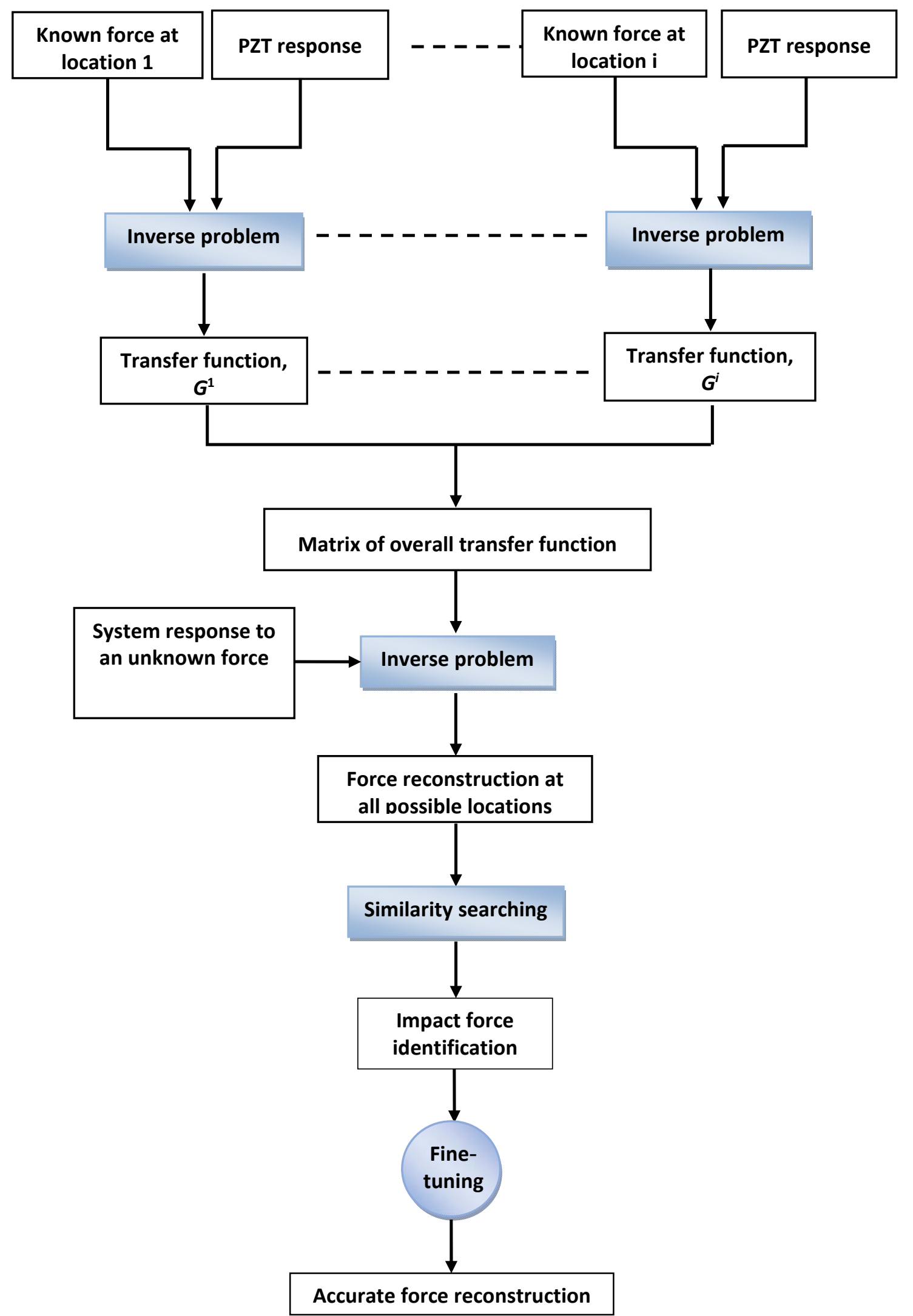

Figure 9. Schematic of impact force identification algorithm using the under-determined approach. 

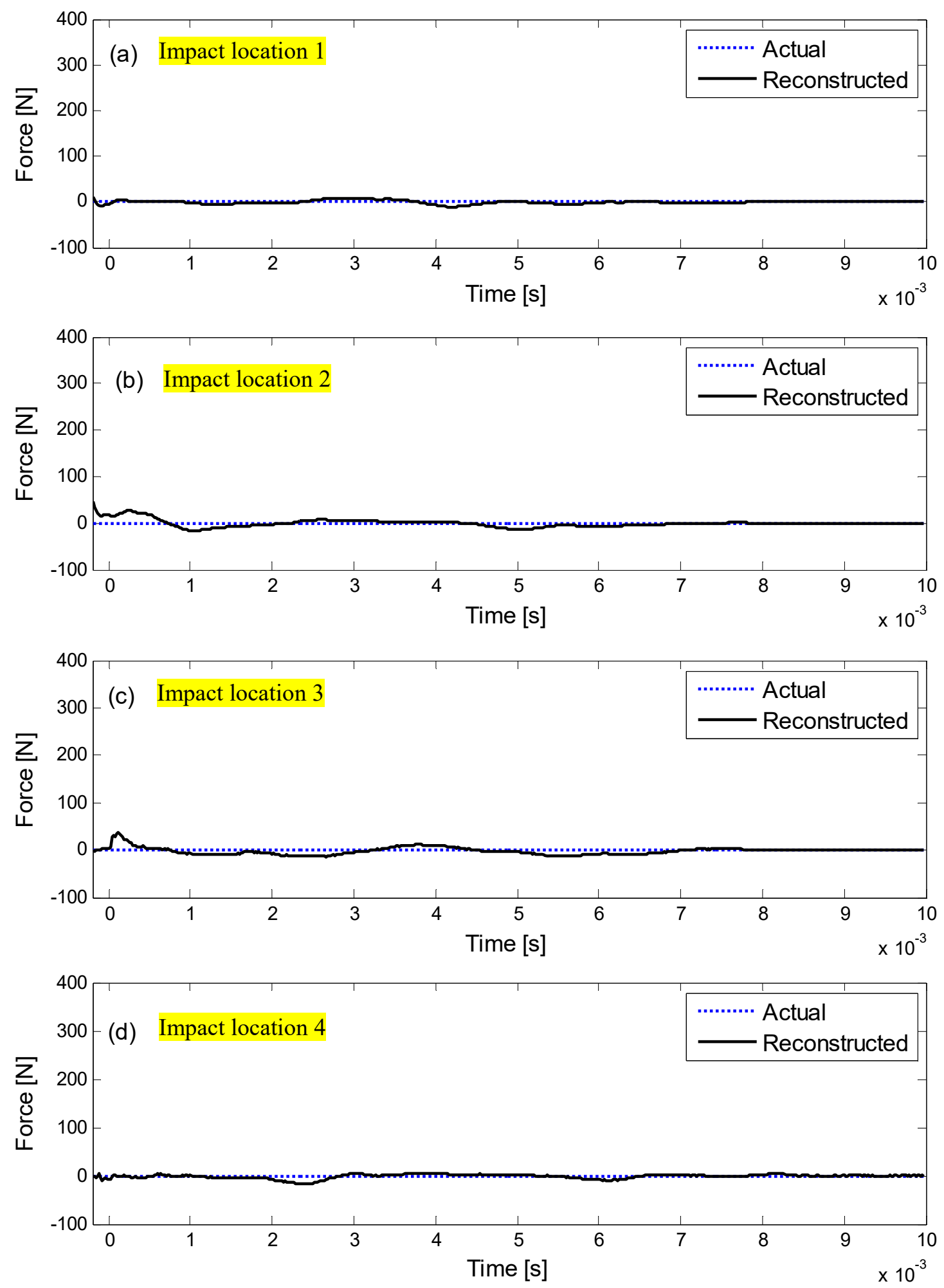

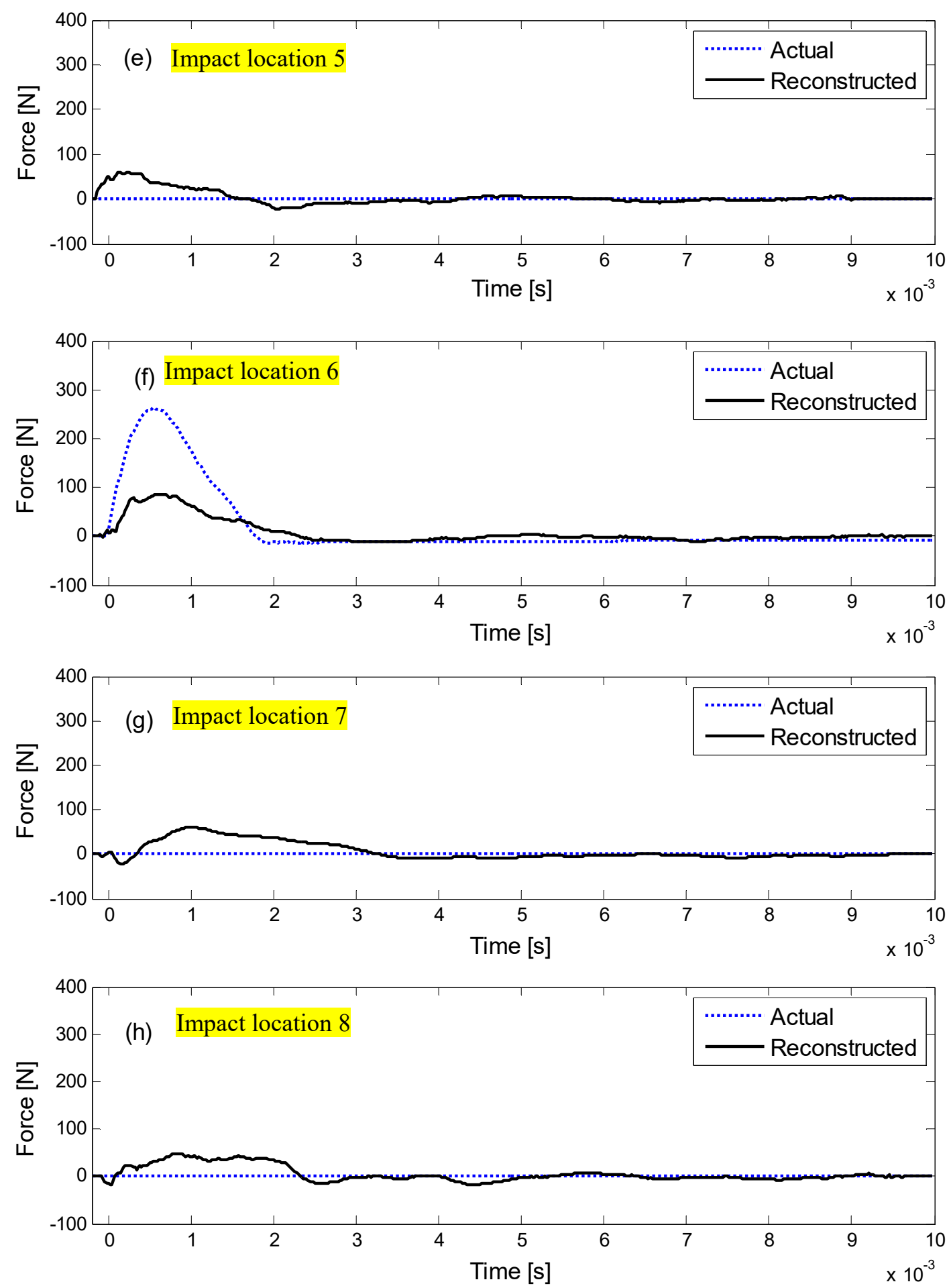

Figure 10. The reconstructed impact forces using the under-determined approach while the true impact location is location 6 . 

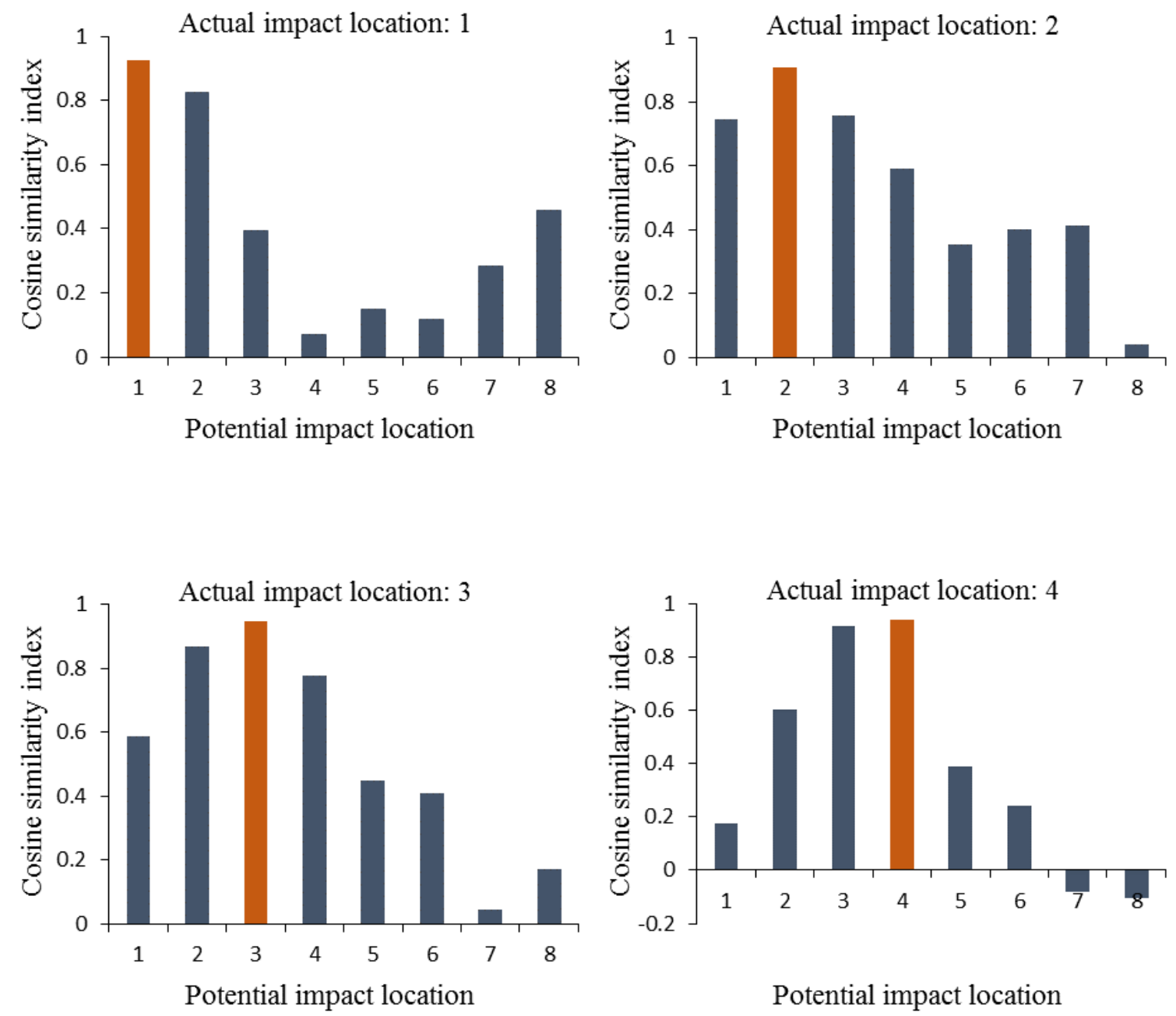


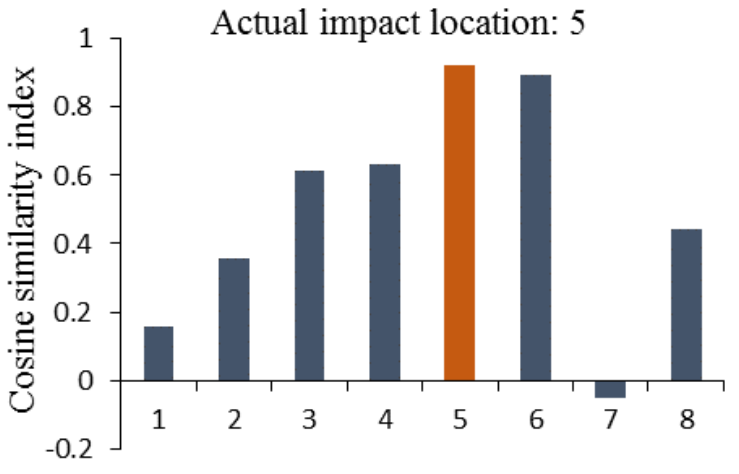

Potential impact location

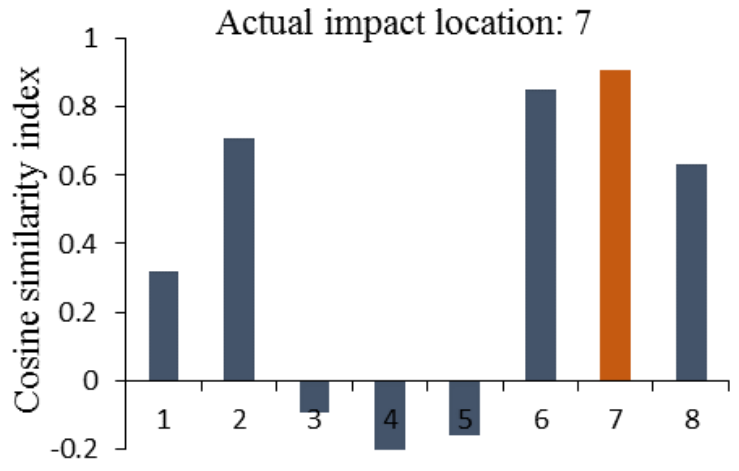

Potential impact location
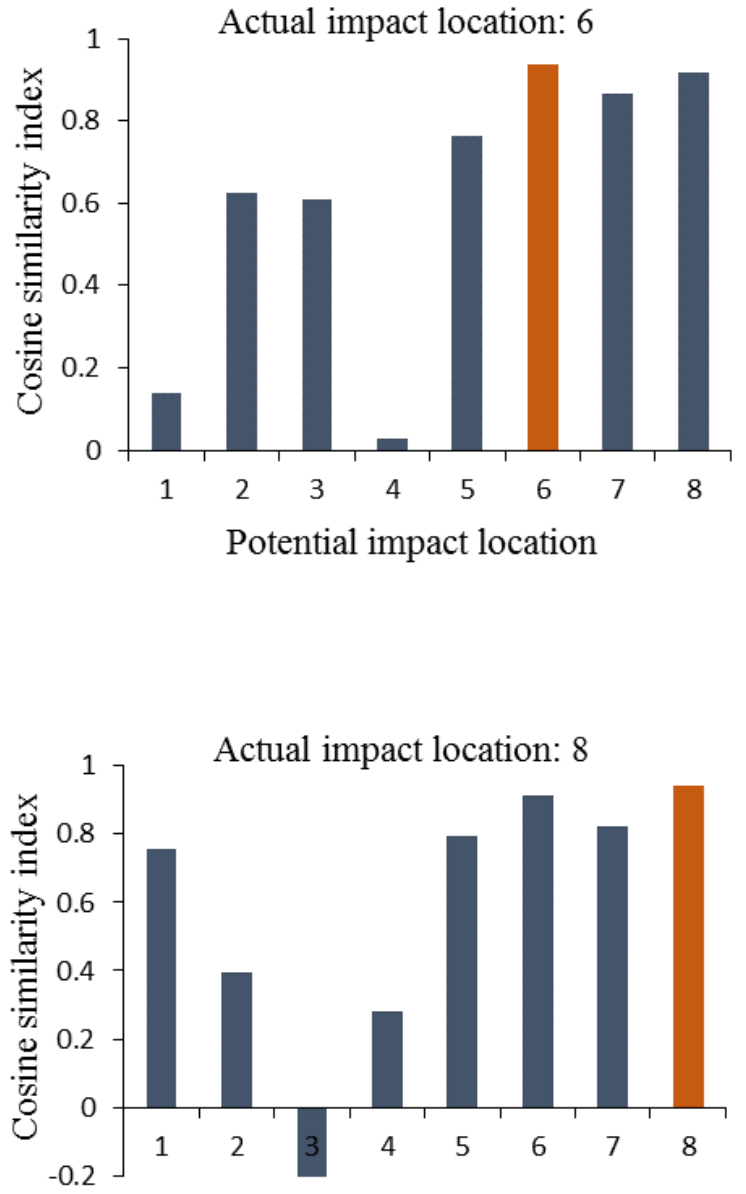

Potential impact location

Figure 11. The cosine similarity indices for the all investigated cases when the impact forces were reconstructed using the under-determined approach. 